\title{
A stochastic model for cell adhesion to the vascular wall
}

\author{
Christèle Etchegaray • Nicolas Meunier
}

Received: date / Accepted: date

\begin{abstract}
This paper analyzes, in the context of cell dynamics in the vicinity of the vascular wall, the stochastic variability of the number of bonds existing between a circulating cell and the wall when there is a negative feedback loop between the cell instantaneous velocity and the bond dynamics: the faster the cell goes, the more likely existing bonds are to disassemble. The goal of this work is to describe the dynamics of the cell according to the blood flow velocity. With a classical birth and death-like dynamics, in the spirit of [?,?], we prove that, under different scaling regimes, the number of bonds, or equivalently the cell velocity, follows either an ordinary differential equation or a stochastic differential equation, whose analysis allow to identify a threshold shear velocity associated with the transition from cell rolling and firm adhesion. Simulations are used to study other aspects of adhesion such as the the mean stopping time of the cell resulting from this dynamics.
\end{abstract}

Keywords Cell adhesion • Metastatic development • Immune response . Atherosclerosis · Stochastic process

1. Introduction

2. A Markovian jump process for the cell adhesion dynamics

3. Continuous limiting models and characterization of the dynamics

4. Discussion

References

Appendices

C. Etchegaray

INRIA Monc, Institut de Mathématiques de Bordeaux, 351, cours de la Libération, 33405 TALENCE, France.

E-mail: christele.etchegaray@inria.fr

N. Meunier

LaMME, CNRS UMR 8071, Université Évry Val d'Essonne, 23 boulevard de France 91037

Évry Cedex, France.

E-mail: nicolas.meunier@univ-evry.fr 


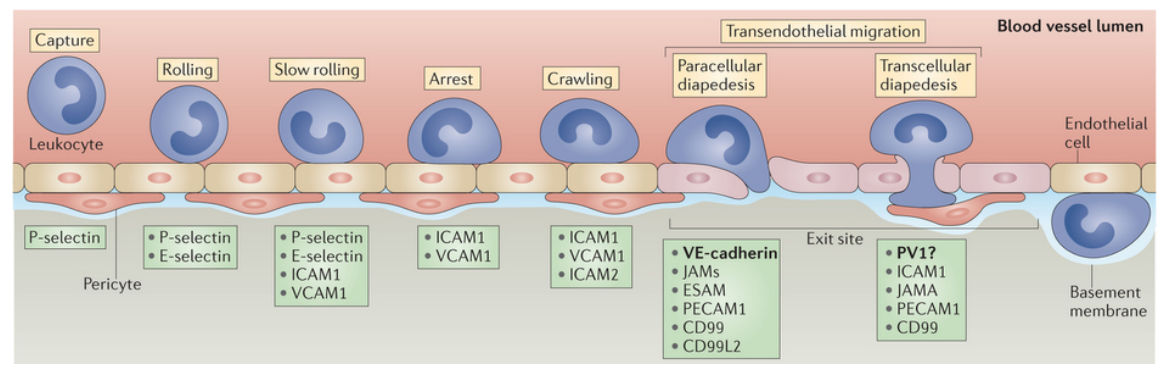

Fig. 1: Scheme of the multistep cascade of leukocyte extravasation. Reprinted by permission from Macmillan Publishers Ltd: Nature Reviews Immunology [?], copyright (2007).

\section{Introduction}

\subsection{Biological context}

Cell adhesion to the vascular wall is a major process involved during inflammation or metastasis invasion [?]. The adhesive interaction between circulating cells and endothelial cells forming the wall occurs in the presence of hemodynamic forces exerted by the blood flow, and is depicted in Figure 1 for leukocytes. Adhesion bonds can form between cell transmembrane proteins called ligands and adhesion receptors at the vascular wall surface. The first step of interaction happens when enough bonds between the cell and the wall are stabilized so that the cell is slowed down, this is the so called capture phase. Then, cells roll along the stimulated endothelium, as new bonds form in the direction of motion and bonds at the back disassemble. This step is mediated by receptor molecules of the selectin family. During rolling, endothelial cells may also be stimulated, and consequently another family of cell receptor molecules, the integrins, is activated on the circulating cell surface. The integrins mediate the firm adhesion which slows the cell enough so that it can penetrate the vascular wall allowing for the further development of the phenomenon at play (immune response, invasion of tissues by metastatic cells e.g, see for example [?]). It is observed that rolling does not always turn to firm adhesion, and cells may return in the blood flow. This shows that cell adhesion to the vascular wall is a complex phenomenon for which mathematical modelling can help understanding its key features.

Cell rolling has been studied in vivo and in vitro in flow chambers, in which isolated cells are rolling on either monolayers of cultured endothelial cells or surfaces coated with selectin or other molecules. It has been observed that the velocities of rolling cells are orders of magnitudes lower than the velocities of non-adherent cells freely moving close to the substratum surface. This indicates adhesive interaction between the rolling cells and the substratum [?].

The rolling motion has been observed to be stochastic both in vivo [?], and in vitro [?]. Variation of the rolling velocities of individual cells in time 
has also been observed for experiments in which they roll on a flat surface bearing a uniform layer of ligands [?]. This suggests that the fluctuations in cells dynamics in the vicinity of the wall is a reflection of the stochastic nature of the cells bond formation. Our work aims at investigating its effect on the cell dynamics in a blood vessel.

\subsection{Existing Models}

There already exists several models and approaches to study cell adhesion on the vascular wall. The simplest approach consists in ignoring the cell spatial structure and to assume a step-wise, stop-and-go motion. In [?] the trajectory of the center of the cell is approximated by a series of rapid steps in between which the cell velocity is zero. Two random variables are used to describe the average distance and lifetime of bond clusters resisting the applied fluid force. Performing some mean field approximation makes it possible to heuristically obtain a Fokker-Planck equation which governs the cell velocity evolution. The drift and diffusion coefficients in the Fokker-Planck equation are heuristically derived from the non smoothed stepping process of cell displacement and expressed in terms of the step size and waiting time of this stepping process. This approach was able to predict that the distribution of rolling velocities is influenced by the analysis of the variance or dispersion of rolling velocity data acquired under different experimental conditions.

In the same spirit, in the absence of fluid flow, macroscopic models have been developed for cell adhesion force [?], where bonds are described as a distribution function. Its dynamics follows a maturation-rupture equation (also called renewal equation). In the limit of large bonds turnover, a macroscopic friction coefficient can be computed [?,?].

In [?], the cell is described as a point carried by the fluid flow and interacting with the endothelium, which is assimilated to a straight line. At the level of the individual receptor molecule, ligand binding and dissociation are stochastic processes (Poisson processes). The bond forces are described by linear elastic forces. In the case where binding and dissociation rates are constant, averaging leads to a deterministic linear Volterra integro-differential equation similar to the one considered in [?,?], that provides information on the cell location. Linear continuous models are not satisfactory as they can not describe the strong dependency of cells arrest on shear flow. In [?] forcedependent bond rates and/or nonlinear elastic laws were also considered. For these nonlinear models a threshold on the blood velocity, under which the cell velocity vanishes, was numerically observed. Furthermore the link between the stochastic nonlinear processes and the deterministic nonlinear equations was only done numerically.

In $[?, ?]$, a numerical model was considered to describe the interplay between hydrodynamic transport and specific adhesion. In these works, the cell is modeled as a hard sphere covered with receptors moving above a planar ligand-bearing wall. The ligand-receptor binding follows a chemical kinetic 
dynamics according to Bell's law, [?]. Bonds then exert elastic forces on the sphere while the linear shear flow exerts both hydrodynamic force and torque. In [?], the Brownian motion of the sphere is taken into account in order to model the spatial receptor-ligand encountering more precisely. Algorithms that allow to simulate the cell motion as well as the formation and rupture of bonds between receptors and ligands are given. The strength of these models is to allow for a numerical study of the influence of bonds that easily rupture in response to force on the motion of the sphere.

\subsection{Results of the paper}

The main goal of this paper is to estimate the effects of the adhesion activity and the blood flow on the dynamics of a circulating cell located in the vicinity of the arterial wall. To do so we build some 1D stochastic models, of birth and death types, for a cell developping adhesive interaction with the vascular wall. The difference in these models lies in the creation, reproduction and dissasembly rates. In the linear case and in the case where there is a nonlinear coupling between at least one of the rates and the instantaneous cell velocity, we study the efficiency of adhesion. For a given blood flow velocity the main characteristics used for the study are the mean number of bonds the cell has created with the wall and the mean time to reach the critical number of bonds that will stop the cell. In particular, we look for a model that is able to capture the transition from cell rolling to either its stopping or its release in the blood flow. For this purpose, we perform and justify some scaling limits to provide key quantities on the effect of the cell adhesion activity on the dynamics of the cell.

Following [?], we consider a minimal discrete stochastic model where the cell is a point particle submitted to blood flow with $1 \mathrm{D}$ constant velocity. The adhesion dynamics consists in a Markovian Jump process for the formation and disassembly of closed bonds related to units of resistive force. The choice of a stochastic model follows biological observations of e.g [?]. This model has some similarities with the model heuristically derived in [?] but takes a more detailed view of the events between the cell and the endothelium in the spirit of [?]. Moreover, the main difference with the model studied in [?] is that the bond force is constant instead of elastic. We model the loop between the cell velocity and the bond formation dynamics. This leads to a nonlinear stochastic jump process to describe the velocity of the cell.

Since the number of bonds involved in cellular adhesion is high enough so that the adhesion dynamics can be considered continuous in time, in a second step we let the number of bonds go to infinity while the contribution of each bond to the adhesion force goes to zero. In the spirit of [?,?], we rigorously derive continuous limiting models for the cellular adhesion dynamics. Depending on the renormalization assumptions, we obtain either a deterministic or a stochastic model, that we both study. The deterministic model successfully predicts the threshold wall shear stress above which rolling does not occur and, 
for some parameter values, it predicts the cell stationary adhesion. We also study the continuous stochastic model and derive information on the mean time needed for the cell to stop. To do so, in a first step we show that in a simplified setting, we obtain the Cox-Ingersoll-Ross (CIR) process, for which the probability density of the arrest time is explicitly given and shows a transition between the stopping of the cell and its release in the blood flow. Then, using a Fokker-Planck approach, we derive the mean stopping time of a circulating cell for the model that includes a feedback of the fluid on the bond dynamics. We believe that this work can have strong implications for the immune response, drug delivery systems, as well as tumor invasion. More precisely, our model could be used as a first step in the construction of a permeability law for the vascular wall. In a further work, it will be compared with experimental in vivo data obtained in [?] in the study of metastatic invasion.

The plan of this article is the following. In Section 2, we detail the construction of the discrete stochastic model for the individual bond dynamics, and we perform its analysis together with numerical simulations. In section 3 , we proceed to rigorous derivations of the continuous equations, either deterministic or stochastic, satisfied by the cell velocity. It allows to analytically study the influence of bonds which have formed between receptors and ligands on the cell motion. In particular, the continuous deterministic model predicts that the cell can either develop no bonds with the vascular wall when the shear velocity is high and/or the wall is in a lowly inflamed state with a low density of adhesion proteins, or the cell decelerates and rolls on the wall with eventually sufficient high decelaration so that the zero velocity is reached. We show that there is a well-defined region of the parameter space where this dichotomy exists and we provide some quantitative information about the cell dynamics. In addition we study the stochastic model and we derive the cell mean stopping time. In section 4 we discuss our results and show that it provides key quantities to characterize the cell's long term motion.

\section{A Markovian Jump process for the cell adhesion dynamics}

We first present the stochastic model used to investigate the adhesion process outlined in the introduction. We will use a classical birth and death model for bonds turnover. Like in most of the literature, see [?], it is assumed that the bond has a duration with an exponential distribution. Then, we study its mathematical properties and we perform some numerical simulations.

\subsection{Modelling approach}

Let us consider a cell carried by the blood flow. We suppose that the size of the gap between the cell and the blood vessel wall is small enough so that bonds between the cell and the vascular wall may always form in the contact area. Since the cell is in the vicinity of the wall, we assume that the blood shear 
flow is $1 \mathrm{D}$, parallel to the vascular wall and with a constant velocity, denoted by $u \in \mathbb{R}_{+}$.

Deformability may play a role in the cell dynamics inside the blood flow and in interaction with the vessel wall. However, some large cells like Circulating Tumor Cells can be very stiff [?], and still show the behaviour under study in this paper. Therefore, we choose here to neglect cell deformability and to focus on the intracellular adhesion dynamics with the vessel wall.

In previous studies, see [?,?] e.g., it was shown that approximating the contact area by a simple geometrical figure (a circle or a rectangle) and neglecting the increase of the contact area with the flow shear rate due to cell deformability do not change qualitatively the analysis. Moreover, as suggested in [?], the cell adhesion is primarily determined by physicochemical properties of adhesion proteins and, thus, to a first approximation, we assume the cell to be a point particle whose position at time $t \geq 0$ is denoted by $X_{t}$.

\section{Velocity model}

To describe the cell motion, we use a non-inertial approximation. Indeed, in the limit of low Reynolds number, viscous forces dominate over inertial forces and the momentum equation reduces to the force balance principle:

$$
V_{t}=u-\gamma F_{t},
$$

where $V_{t} \in \mathbb{R}$ is the cell velocity, $u$ is the blood shear flow and the cell is subjected to a macroscopic resistive force, denoted by $F_{t} \in \mathbb{R}_{+}$, induced by the bonds that contribute to decelerating the cell, see Figure 1. The parameter $\gamma$ is such that $\gamma^{-1}$ is a friction coefficient, following a linear force-velocity relation. The previous equation is valid only for $\gamma F_{t} \leq u$, as for a maximal force the cell stops, and the model is no longer valid. The resistive force arises from the strength of the cell adhesion to the vessel wall. Cellular adhesion is a macroscopic readout of the forces exerted by the wall on the cell through each bond [?]. As a consequence, we assume that

$$
F_{t}=f N_{t},
$$

where $f \geq 0$ is the typical force generated by a stabilized bond, and $N_{t}$ is the number of stabilized bonds at time $t$. Note that $f$ ranges in $\mathrm{pN}$ [?], but its precise value depends on the experimental conditions.

Non-dimensionalization

We introduce now typical quantities for our problem: we use the typical force related to a bond, the corresponding velocity, and the typical range of lifetime of a bond [?]. We obtain the following table:

$$
\begin{array}{c|c|c}
\bar{F} & \bar{V}=\gamma \bar{F} & \bar{T} \\
\hline f & \gamma f & 1 \mathrm{~s}
\end{array}
$$


Therefore, keeping the same notations for simplicity, the nondimensionalized problem writes

$$
V_{t}=u-N_{t} .
$$

We now construct the process $\left(N_{t}\right)_{t}$ of the number of stabilized bonds over time.

Stochastic model for the adhesive force

Let us now present the simple discrete model we use to describe the individual bonds dynamics. We write $\left(N_{t}\right)_{t}$ the Markovian processes for the number of stabilized bonds at time $t$, that follows a classical birth and death-like dynamics in the state space $\mathbb{N}$.

- New bonds form spontaneously at rate $c(u)=c \mathbb{1}_{u \leq u^{*}}$, for a velocity treshold $u^{*}$ above which no new bonds can be created, due to the high blood velocity.

- Each existing bond can reproduce at constant rate $r$. This phenomenon captures the local reinforcement of the connection to the vessel wall by implication of integrins in adhesion growth, that can be imputed to cytoskeletal forces or external stresses [?]. Moreover, intuitively, if an adhesion complex is composed of a large number of bonds, the unbounded molecules can find an attachment more easily compared to a less stable adhesion formed of fewer molecules.

- Each bond dissociates at the velocity-dependent rate $d\left(V_{t}\right)=d e^{\alpha V_{t}}=$ $d e^{\alpha\left(u-N_{t}\right)}$. We choose here an exponential relation, where $d$ is the unstressed bonds dissociation rate, and $\alpha$ is a sensitivity parameter. This choice accounts for the fact that the average lifetime of an adhesion site changes with the applied tension from the blood flow so that the faster the cell goes, the more likely existing bonds are to disassemble. Note that since the cell velocity is bounded by $u$ in our study, so is the dissociation rate. In the following, we will write indifferently $d\left(V_{t}\right)$ or $d\left(N_{t}\right)$.

Remark 1 The rate for a single bond formation between two proteins is actually mostly determined by the time the two proteins spend near one another. Therefore, the rate $c$ should depend on the cell velocity when the relative velocity between the cell surface and the wall is non zero. A more realistic choice for $c$ would be to consider a decreasing function of the instantaneous cell velocity $V_{t}$. A prototypical behaviour would be given by $c(v)=\left(u_{*}-v\right)_{+}$ where $(\cdot)_{+}$denotes the positive part function. Recalling that $V_{t}$ is related to $N_{t}$ this rewrites $c(n)=\left(u_{*}-u+n\right)_{+}$for $n$ the number of stabilized bonds. For such a choice, and assuming that $v \leq u_{*}$, the dependence on $n$ then only provides an additional contribution to the reproduction rate.

Note that these rates are also representative of the adhesive properties of the endothelial cells forming the vessel wall. The key point here is that there is a feedback loop between the instantaneous cell velocity and the bonds dynamics. 
More elaborate dependency could be considered (see e.g [?,?]), in particular involving age dependences to model the bond elasticity (see [?]), but we choose to keep a minimal set of parameters, as for simplicity as for the sake of clarity.

The balance between the adhesion force on the one hand, and the load and torque created by the blood flow on the other hand then determines the outcome of the dynamics: cell rolling and arrest or its release in the blood flow.

\subsection{Mathematical properties of the discrete model}

In this section we derive some mathematical properties and we perform numerical simulations on the process $\left(N_{t}\right)_{t}$. The dissociation rate being nonlinear, classical tools do not apply. Since we are interested in the dynamics while $V_{t} \geq 0$, that is to say while $N_{t} \leq u$, we define the stopping time

$$
\tau_{u}:=\inf _{t \geq 0}\left\{N_{t} \geq u\right\} .
$$

We are interested in the Markovian jump process $\left(N_{t}\right)_{t \in\left[0, \tau_{u}\right]}$ defined by the following transitions:

$$
n \mapsto\left\{\begin{array}{l}
n+1 \text { at rate } \lambda(n)=c(u)+r n, \\
n-1 \text { at rate } \mu(n)=d(n) n,
\end{array}\right.
$$

where $\lambda$ and $\mu$ are defined on $\mathbb{N}$, and are bounded. It is classical that such a process is well-defined (see e.g [?]). We also control the mean number of bonds in finite time.

Proposition 1 (Moments propagation) Assume that there exists $p \geq 1$ such that $\mathbb{E}\left[N_{0}^{p}\right]<+\infty$. Then,

$$
\mathbb{E}\left[\sup _{t \in\left[0, T \wedge \tau_{u}\right]} N_{t}^{p}\right]<+\infty \forall T>0 .
$$

Proof This Proposition is proved in a more general framework in Appendix A

The mean path of this process can not be fully studied in the general case. Indeed, for $\mathbb{E}\left[N_{0}\right]<+\infty$, we classically write the mean equation

$$
\mathbb{E}\left[N_{t \wedge \tau_{u}}\right]=\mathbb{E}\left[N_{0}\right]+c(u) \mathbb{R}\left[t \wedge \tau_{u}\right]+\mathbb{E}\left[\int_{0}^{t \wedge \tau_{u}}\left(r-d e^{\alpha\left(u-N_{s}\right)}\right) N_{s} \mathrm{~d} s\right]
$$

where even assuming $t \leq \tau_{u}$, the nonlinearity would prevent any analysis.

In a simpler case, namely when there is no feedback of the cell velocity on the adhesion dynamics, and without considering the stopping time $\tau_{u}$, we obtain a classical immigration-birth-death process, that was already studied in [?,?]. More precisely, $\left(N_{t}\right)_{t}$ then follows a negative binomial distribution of parameters $\left(\frac{c(u)}{r}, \frac{r}{d}\right)$. It follows that 


$$
\mathbb{E}\left[N_{t}\right]= \begin{cases}\mathbb{E}\left[N_{0}\right]+c(u) t & \text { if } r=d, \\ \mathbb{E}\left[N_{0}\right] e^{(r-d) t}+\frac{c(u)}{r-d}\left(e^{(r-d) t}-1\right) & \text { otherwise. }\end{cases}
$$

At steady state, one finds

$$
\mathbb{E}[N]_{\infty}:=\left\{\begin{array}{ll}
\frac{c(u)}{d-r} & \text { for } r<d, \\
+\infty & \text { otherwise, }
\end{array} \Leftrightarrow \mathbb{E}[V]_{\infty}:= \begin{cases}u-\frac{c(u)}{d-r} & \text { for } r<d \\
-\infty & \text { otherwise }\end{cases}\right.
$$

with

$$
\operatorname{Var}\left(N_{\infty}\right)=\frac{c(u)}{d\left(1-\frac{r}{d}\right)^{2}}
$$

In the case of a circulating cell, we are only interested in the situation where $v \leq u$. As a consequence, assuming $\mathbb{E}\left[N_{0}\right]=0$ and $u>0$, we obtain the following mean asymptotic behaviours:

\begin{tabular}{l|c||c|c}
$\begin{array}{l}u>u_{*} \\
c(u)=0\end{array}$ & \multicolumn{1}{|c}{$v=u$} & Cell release \\
\hline \hline $\begin{array}{l}u \leq u_{*} \\
c(u)=c>0\end{array}$ & $r<d$ & $0<\frac{c}{d-r}<u$ & Cell rolling \\
\cline { 3 - 4 } & & $0<u \leq \frac{c}{d-r}$ & Cell arrest \\
\cline { 2 - 4 } & $r \geq d$ & & Cell arrest
\end{tabular}

These results show that the birth and death dynamics without feedback intrinsiquely carries a dichotomic asymptotic behaviour. However, such a model is not fully satisfactory, since no shear treshold effect appears further from the one ensuring the initiation of the adhesive interaction.

\subsection{Numerical Simulations}

The process $\left(N_{t}\right)_{t}$ being Markovian, it can be simulated directly events after events. Consider the population size $N_{T_{k}}$ at time $T_{k}$. Then,

- the global jump rate writes $\varsigma_{k}=\lambda\left(N_{T_{k}}\right)+\mu\left(N_{T_{k}}\right)$. This means that the time before the next event is a random variable distributed following an exponential law of parameter $\varsigma_{k}$. A realization of this law provides $T_{k+1}$.

- a new bond is created with probability $\frac{\lambda\left(N_{T_{k}}\right)}{\varsigma_{k}}$, while with probability $\frac{\mu\left(N_{T_{k}}\right)}{\varsigma_{k}}$ a randomly chosen bond disassembles, and $N_{T_{k+1}}$ follows.

This procedure can be iterated to give the time evolution of the process. Numerical simulations of the process are displayed in Figure 2. It is observed that the velocity may either shrink to zero or remain close to $u$ for the same parameter values. Note also that rolling phases are observed in both cases. 


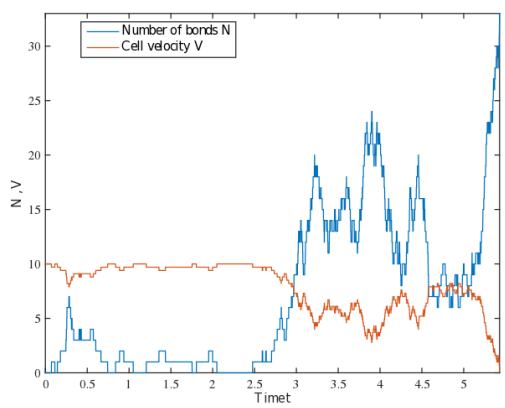

(a) The stochasticity induces a large rolling phase that ends up in the cell arrest.

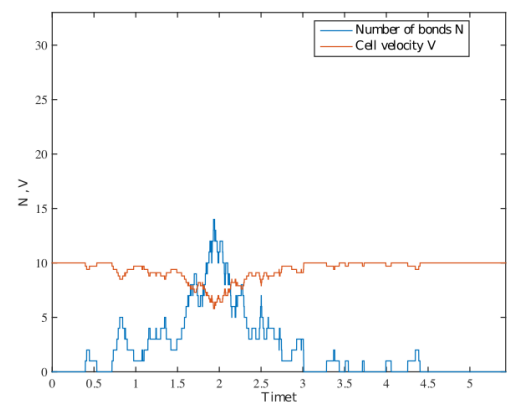

(b) The cell only experiences a small rolling phase that does not prevent its release in the blood flow.

Fig. 2: Numerical simulations of the discrete process defined by (1). Parameters: $(u, c, r, d, \alpha)=(33,4,5,3,0.1)$.

\section{Continuous limiting models and characterization of the dynamics}

It has been seen in the previous section that, for the nonlinear model, the mean path of this process can not be fully studied in the general case. In this section we separate the scale of the adhesion dynamics from the one of the cell motion. Such scale separation is justified by the large number of bonds, and by the very fast binding dynamics, compared to the cell displacement. This is illustrated by the fact that the lifetime of a bond is of the order of $1 \mathrm{~s}$, the ligands association rate is about $10^{3} \mathrm{~s}^{-1}$ whereas the cell rolling velocity is around $30 \mu \mathrm{m} \mathrm{s}^{-1}$, see [?] and the references therein. As will be seen, this assumption allows to use a scaling approach to derive two continuous limiting models, for which deeper analysis can be pursued.

More precisely, we let the number of bonds grow to infinity while the individual contribution to the adhesion force shrinks to zero, so that the global adhesive force keeps a constant range. We also accelerate the dynamics of bonds turnover using two scalings, that lead to different types of limiting continuous models.

Let $K \geq 1$ be a parameter scaling the number of the discrete adhesions we consider. We assume that $\frac{1}{K}$ scales the force generated by each one. This amounts to looking at adhesion sites at a smaller and smaller scale. Moreover, we assume that the dynamics gets faster and faster. Hence, we consider now $K$-dependent rates $c^{K}, r^{K}$, and $d^{K}$, related to the process $\left(N_{t}^{K}\right)_{t}$. We define the renormalized process $\left(Z_{t}^{K}\right)_{t}$ by

$$
Z_{t}^{K}=\frac{1}{K} N_{t}^{K} \in \frac{1}{K} \mathbb{N}
$$


3.1 Deterministic continuous limiting model

We consider the following rates:

$$
c^{K}(u)=K c(u), \quad r^{K}=r \quad \text { and } d^{K}\left(K Z_{t}^{K}\right)=d\left(Z_{t}^{K}\right) .
$$

In other words, while we consider an increasing number of smaller adhesions, only their spontaneous formation is intensified, while the self-enhancement of the adhesion dynamics and their lifetime stay the same. From the modelling viewpoint such an assumption amounts to considering an increasing number of smaller adhesions, each of them involving a small number of proteins on each side (wall and cell). Note that since the reproduction is not accelerated, the clustering leading to adhesion growth is not large enough to induce stochastic fluctuations at the cell level. The case where it does is considered hereafter, see paragraph 3.2 , when the individual dynamics of the bonds is also accelerated. In this context, we obtain the following convergence result.

Theorem 1 If $Z_{0}^{K} \underset{K \rightarrow+\infty}{\longrightarrow} n_{0} \in \mathbb{R}_{+}$in probability, and if

$$
\sup _{K>0} \mathbb{E}\left[\left(Z_{0}^{K}\right)^{2}\right]<+\infty
$$

then, for $T>0,\left(Z^{K}\right)_{K>0}$ converges in law in $\mathbb{D}\left([0, T], \mathbb{R}_{+}\right)$to the unique continuous function $n \in \mathcal{C}\left([0, T], \mathbb{R}_{+}\right)$solution to

$$
n(t)=n_{0}+\int_{0}^{t} c(u)+(r-d(n(s))) n(s) \mathrm{d} s .
$$

Remark 2 By the Gronwall lemma, one has for $T<\infty$,

$$
\sup _{t \in[0, T]} n(t) \leq\left(n_{0}+c T\right) e^{r T}<+\infty,
$$

showing that the global density stays finite in finite time.

Proof The proof is displayed in Appendix B.

We perform now the analysis of the limiting problem. Let us define the function $F$ by: $F(n)=c \mathbb{1}_{u \leq u_{*}}+\left(r-d e^{\alpha(u-n)}\right) n$. We prove the following result.

Proposition 2 Assume that the rates are given by (5). Then the stationary state(s) $n^{\infty}$ of (6) are as follows.

1. If $u>u_{*}$, then the system admits two stationary states $n_{1}^{\infty}=0$ and $n_{2}^{\infty}=u-\frac{1}{\alpha} \ln \left(\frac{r}{d}\right)$. The smallest is stable and the largest is unstable.

2. If $u \leq u_{*}$,

(a) for $u \leq \frac{1}{\alpha} \ln \left(\frac{r}{d}\right)$, then $n^{\infty}=+\infty$.

(b) for $u>\frac{1}{\alpha} \ln \left(\frac{r}{d}\right)$, then there exists a unique $0<\bar{n}<\frac{1}{\alpha}$ such that $F^{\prime}(\bar{n})=0$ and

i. If $F(\bar{n})>0$, then $n^{\infty}=+\infty$. 
ii. If $F(\bar{n})=0$, then $\bar{n}$ is the unique stationary solution.

iii. If $F(\bar{n})<0$, then there exists two stationary solutions $n_{1}^{\infty}$ and $n_{2}^{\infty}$, such that $0<n_{1}^{\infty}<\bar{n}<n_{2}^{\infty}<+\infty$, the smallest being stable and the largest unstable.

Proof The case $u>u_{*}$ follows from a direct computation. Consider the case where $u \leq u_{*}$, then one has

$$
n^{\prime}(t)=c+\left(r-d e^{\alpha(u-n(t))}\right) n(t)=F(n(t)) .
$$

A quick computation shows that

$$
\begin{aligned}
& F^{\prime}(n)=r+d(\alpha n-1) e^{\alpha(u-n)}, \\
& F^{\prime \prime}(n)=\alpha d(2-\alpha n) e^{\alpha(u-n)} .
\end{aligned}
$$

We can study the sign of $F^{\prime}(n)$ and get the following variation table:

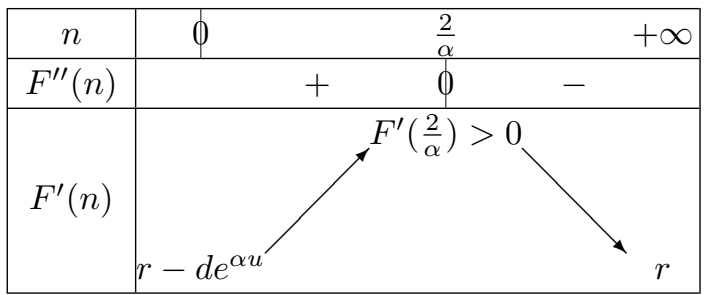

As a consequence,

- If $u \leq \frac{1}{\alpha} \ln \left(\frac{r}{d}\right)$, then $\forall n \in \mathbb{R}_{+}, F(n) \geq c>0$, hence $n^{\infty}=+\infty$.

- If $u>\frac{1}{\alpha} \ln \left(\frac{r}{d}\right)$, then there exists a unique $\bar{n}>0$ such that $F^{\prime}(\bar{n})=0$. Since $\bar{n}<\frac{2}{\alpha}$, we obtain the following variation table from which the result follows:

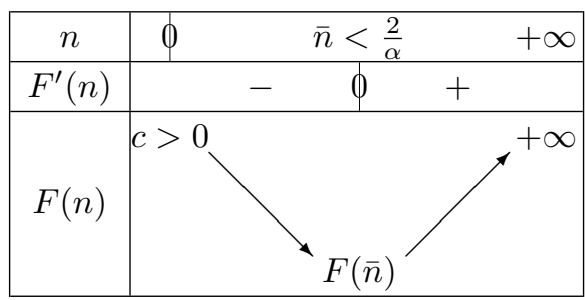

Notice that since $u>\frac{1}{\alpha} \ln \left(\frac{r}{d}\right) \Leftrightarrow d e^{\alpha u}>r$, both behaviours arise according to the comparison between the reproduction and death rates. Note also that since $F^{\prime}$ is strictly increasing on $\left(0, \frac{2}{\alpha}\right)$ and that $F^{\prime}(1 / \alpha)=r>0$, we have that $\bar{n}<1 / \alpha$.

Let us comment on these results. First of all, not surprisingly, our model successfully predicts the threshold wall shear stress above which nor capture nor rolling does occur. This is due to the regulation by shear of the number of bonds: the number of bonds falls below one. Moreover, the model predicts existence of cell adhesion bistability, which results from the competition between 
the two processes taking place in the cell-wall contact area: bond formation and rupture. Finally, the model predicts stationary adhesion which is observed experimentally.

Remark 3 Note that if $u_{*} \geq u>\frac{1}{\alpha} \ln \left(\frac{r}{d}\right)$ the three cases described in the proposition above may occur. Indeed, consider the particular case where $d>r$ and $u=\frac{1-\frac{r}{d}}{\alpha}$, then $\bar{n}=u$ and $F(\bar{n})=c-\frac{(d-r)^{2}}{\alpha d}$ whose sign depends on the value of $c$. The dynamics is then dependent on the ability of the cell to form bonds at primary contact.

We are now interested in the consequences of this study for our model of cell adhesion to the vessel wall. Therefore we consider that the problem (6) is valid up until the adhesion density reaches $u$, in which case the cell velocity reaches 0 . The following Corollary locates $u$ with respect to the stationary state(s) of the system. This allows us to assess the cell fate depending on the parameter values. For $\alpha>0$, denote the key parameters

$U_{\alpha}:=\frac{1}{\alpha} \ln (r / d) \quad \bar{U}_{\alpha}:=\frac{1}{\alpha}(1-r / d) \quad U_{c}:=\frac{c}{d-r} \quad \bar{C}:=\frac{1}{\alpha d}(r-d)^{2}$.

Corollary 1 Let $n_{0} \in[0, u]$ with $u>0$. Then, the problem (6) admits either one stationary state denoted by $n^{\infty}$, or two stationary states $n_{1,2}^{\infty}$ such that $0<n_{1}^{\infty}<n_{2}^{\infty}$, the smaller one being stable and the larger unstable.

1. If $u>u_{*}$ :

(a) for $r \leq d$, then $n_{1}^{\infty}=0$ and $n_{2}^{\infty}=u-U_{\alpha} \geq u$.

(b) for $r>d$, if $u \leq U_{\alpha}, n^{\infty}=0$; if $u>U_{\alpha}$, one has $n_{1}^{\infty}=0<n_{2}^{\infty}=$ $u-U_{\alpha}<u$.

2. If $u \leq u_{*}$, then

(a) for $r<d, \exists$ ! $0<\bar{n}<\frac{1}{\alpha}$ such that $F^{\prime}(\bar{n})=0$.

$i$. For $u>\bar{U}_{\alpha}, F^{\prime}(u)>0$, so that $u>\bar{n}$.

A. If $u>U_{c}$, then we have $0<n_{1}^{\infty}<\bar{n}<u<n_{2}^{\infty}$.

$B$. If $u=U_{c}$, then $0<n_{1}^{\infty}<n_{2}^{\infty}=u$.

C. If $u<U_{c}$, then if $F(\bar{n})<0$, one has $0<n_{1}^{\infty}<\bar{n}<n_{2}^{\infty}<u$, if $F(\bar{n})=0$, then $n^{\infty}=\bar{n}<u$; if $F(\bar{n})>0$, then $n^{\infty}=+\infty$.

ii. For $u=\bar{U}_{\alpha}$, then $u=\bar{n}$.

A. If $c>\bar{C}$, then $n^{\infty}=+\infty$.

$B$. If $c=\bar{C}$, then $n^{\infty}=u$.

C. If $c<\bar{C}$, then $0<n_{1}^{\infty}<u<n_{2}^{\infty}$.

iii. For $u<\bar{U}_{\alpha}$, then $0<u<\bar{n}$.

A. If $u=U_{c}$, then $n_{1}^{\infty}=u<n_{2}^{\infty}$.

B. Otherwise, if $F(\bar{n})=0$, then $u<n^{\infty}=\bar{n}$; if $F(\bar{n})>0$, then $n^{\infty}=+\infty$. Finally, if $F(\bar{n})<0$, then $u<n_{1}^{\infty}<n_{2}^{\infty}$ when $u<U_{c}$, and $n_{1}^{\infty}<u<\bar{n}$ when $u>U_{c}$.

(b) For $r=d$, there exists a unique $0<\bar{n}<\frac{1}{\alpha}$ such that $F^{\prime}(\bar{n})=0$ and $u>\bar{n}$. If $F(\bar{n})<0$, we have $0<n_{1}^{\infty}<\bar{n}<n_{2}^{\infty}<u$. If $F(\bar{n})=0$, $n^{\infty}=\bar{n}<u$. If $F(\bar{n})>0, n^{\infty}=+\infty$.

(c) For $r>d, F(u)>0$. 
i. if $u \leq U_{\alpha}$, then $n^{\infty}=+\infty$

ii. if $u>U_{\alpha}$, then $\exists ! 0<\bar{n}<\frac{1}{\alpha}$ such that $F^{\prime}(\bar{n})=0$ and $u>\bar{n}$. Then, if $F(\bar{n})<0$, we have $0<n_{1}^{\infty}<\bar{n}<n_{2}^{\infty}<u$. If $F(\bar{n})=0$, $n^{\infty}=\bar{n}<u$. If $F(\bar{n})>0, n^{\infty}=+\infty$.

Proof The corollary results from Proposition 2 combined with the sign analysis of $F(u)$ and $F^{\prime}(u)$ that depend on parameters values.

Let us give an interpretation of these situations. Table $3 \mathrm{a}$ gives the outcome of the cell dynamics in the case when $u>u_{*}$. In this case, the blood flow is so fast that the cell does not initiate any new adhesion. However, the dynamics may still be interesting in the case where some bonds already exist and may be stabilized by the self-enhancement of the activity of formation of bonds. Then, if $r \leq d$, dissociation is always more frequent than enhancement of existing adhesions, so that starting with $n_{0} \leq u$, the cell tends to be released in the blood flow. When $r>d$, then depending on $\alpha$, the sensitivity of the dissociation rate on the cell velocity, the cell is either released in the blood flow, or may experience rolling when $\alpha$ is small.

Table $3 \mathrm{~b}$ shows the cell possible outcomes in the case when $u \leq u_{*}$. In this situation, the whole adhesion formation dynamics is active. Then, the conditions discriminating between different cell fates are based on the balance between the formation of adhesion bonds (related to $r$ and $c$ ), and their dissolution (related to $d$ and $\alpha$ ). This study shows how Equation (6) carries more complex behaviours in comparison with the mean linear ODE (3).

\begin{tabular}{c|l||l|r}
$r>d$ & $\begin{array}{l}u \leq U_{\alpha} \\
u>U_{\alpha}\end{array}$ & $n^{\infty}=0$ & $n_{1}^{\infty}=0<n_{2}^{\infty}=u-U_{\alpha}<u$ \\
& \multicolumn{1}{c|}{ Cell release } \\
\multicolumn{2}{c|}{ Cell release and Cell rolling }
\end{tabular}

(a) Case $u>u_{*}$

\begin{tabular}{|c|c|c|c|c|}
\hline \multirow[t]{2}{*}{$r>d$} & \multirow{2}{*}{\multicolumn{2}{|c|}{$\begin{array}{l}u \leq U_{\alpha} \\
u>U_{\alpha}\end{array}$}} & $n^{\infty}=+\infty$ & \multirow{4}{*}{$\begin{array}{r}\text { Cell arrest } \\
\text { Cell arrest } \\
\text { or Cell rolling } \\
\text { or Two rolling regimes }\end{array}$} \\
\hline & & & \multirow{3}{*}{$u>\bar{n}$} & \\
\hline \multicolumn{3}{|c|}{$r=d$} & & \\
\hline \multirow[t]{7}{*}{$r<d$} & \multirow{3}{*}{$u>\bar{U}_{\alpha}$} & $u<U_{c}$ & & \\
\hline & & $u=U_{c}$ & $0<n_{1}^{\infty}<n_{2}^{\infty}<u$ & Two rolling regimes \\
\hline & & $u>U_{c}$ & \multirow{2}{*}{$0<n_{1}^{\infty}<u<n_{2}^{\infty}$} & \multirow{2}{*}{ Cell rolling and Cell arrest } \\
\hline & \multirow[b]{2}{*}{$u=\bar{U}_{\alpha}$} & $c<\bar{C}$ & & \\
\hline & & $\begin{array}{l}c=\bar{C} \\
c>\bar{C}\end{array}$ & $\begin{array}{l}n^{\infty}=u \\
n^{\infty}=+\infty\end{array}$ & \multirow[t]{2}{*}{ Cell arrest } \\
\hline & \multirow[b]{2}{*}{$u<\bar{U}_{\alpha}$} & $u=U_{c}$ & $0<n_{1}^{\infty}=u<n_{2}^{\infty}$ & \\
\hline & & $u \neq U_{c}$ & $0<u<\bar{n}$ & $\begin{array}{r}\text { Cell rolling and Cell arrest } \\
\text { or Cell arrest }\end{array}$ \\
\hline
\end{tabular}

(b) Case $u \leq u_{*}$

Fig. 3: Table of stationary solutions of $\sqrt{6}$ and corresponding situations. Stationary solutions are in red if unstable, blue if stable. 
3.2 Stochastic continuous limiting model

In this section, we consider the following rates:

$$
c^{K}(u)=K c(u) \quad r^{K}=r+K a \quad d^{K}\left(K Z_{t}^{K}\right)=d\left(Z_{t}^{K}\right)+K a,
$$

with $K>0$ and $a>0$. The whole adhesion dynamics is therefore accelerated. Note that the same acceleration for reproduction and death permits to keep the same bounded individual growth rate $r^{K}-d^{K}=r-d$. This way, even if each adhesion bond reproduces and dies infinitely faster, its contribution to the global adhesion growth remains the same.

Theorem 2 If for $K \rightarrow+\infty$ the initial value $Z_{0}^{K}$ converges in law to a $\mathbb{R}_{+}$valued random variable $N_{0}$, with

$$
\sup _{K>0} \mathbb{E}\left[\left(Z_{0}^{K}\right)^{2}\right]<+\infty
$$

then $\left(Z^{K}\right)_{K>0}$ converges in law in $\mathbb{D}\left([0, T], \mathbb{R}_{+}\right)$to the continuous process $N=\left(N_{t}\right)_{t \in[0, T]} \in \mathcal{C}\left([0, T], \mathbb{R}_{+}\right)$solution of

$$
\mathrm{d} N_{t}=b\left(N_{t}\right) \mathrm{d} t+\sigma\left(N_{t}\right) \mathrm{d} B_{t}
$$

with $B_{t}$ a Brownian Motion, $b\left(N_{t}\right)=c(u)+\left(r-d\left(N_{t}\right)\right) N_{t}$ and $\sigma\left(N_{t}\right)=\sqrt{2 a N_{t}}$.

Proof The proof is displayed in Appendix C.

Remark 4 The solution to the SDE (7) is almost surely positive if $b(n) \geq 0$ for all $n \geq 0$, and for a positive initial state (see the 1D comparison principle in [?] e.g.).

Numerical simulations We performed numerical simulations of the SDE (7), using a symmetrized Euler scheme in order to preserve the positivity of the process. It consists in taking the absolute value of the classical Euler scheme (see e.g [?]). More precisely, the scheme is the following: write $\left(N_{k}\right)_{k}$ for the discretization of $\left(N_{t}\right)_{t}$, where $N_{k}$ corresponding to the time $t_{k}=k \Delta t$. Then, define $N_{0}=n_{0}$ and for $k \geq 0$,

$$
N_{k+1}=\left|N_{k}+b\left(N_{k}\right) \Delta t+\sqrt{2 a \Delta t N_{k}} W\right|,
$$

with $W \sim \mathcal{N}(0,1)$. It is proved in [?] that strong $L^{1}$ convergence holds for this scheme if

$$
\frac{\sigma^{2}}{8}\left(\frac{2 b(0)}{\sigma^{2}}-1\right)^{2}>3 P \vee 4 \sigma^{2},
$$

for $P \geq|r-d|$ and $\Delta t \leq \frac{1}{2 P}$. This condition allows to deal with the non Lipschitz diffusion coefficient, and rewrites in our case

$$
\frac{a}{4}\left(\frac{c}{a}-1\right)^{2}>(3 P \vee 8 a)
$$

As an example, $\frac{a}{4}\left(\frac{c}{a}-1\right)^{2}>8 a$ is equivalent to $\left(\frac{c}{a}-1\right)^{2}>32$, that is verified for $c>7 a$. The numerical simulations are displayed in Figure 4 , and show two typical arrest and release situations. 


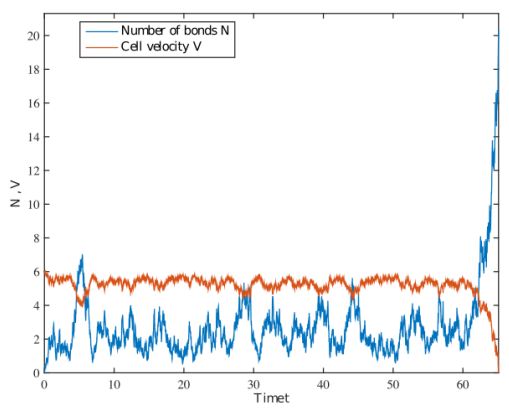

(a) Example of cell arrest, $c=4$.

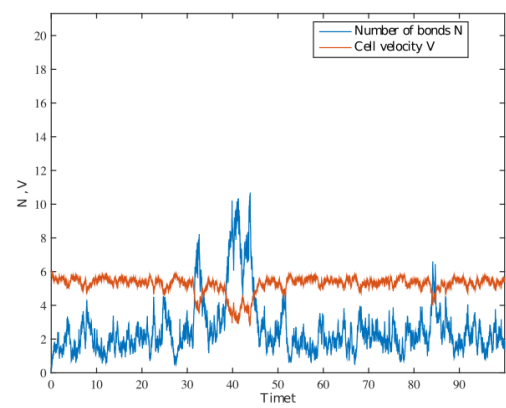

(b) Example of cell rolling, $c=5$.

Fig. 4: Numerical simulations of the solution of the SDE (7). Parameters: $(u, r, d, \alpha, a)=(20,5,4,0.1,0.55)$.

\section{Stopping time}

The rolling motion of individual cells has been observed to fluctuate randomly both in vivo and in vitro, hence it is natural to study the stochastic model obtained in Theorem 2 to understand the effect of these fluctuations. In particular we are interested in the probability for a rolling cell to stop, that is to say for the velocity to reach 0 . More precisely, in this section we compute the mean time $\tau_{u}$ needed for the process to reach $u$ starting from $n_{0}$.

The case without feedback: the CIR process When we assume that the cell velocity exerts no feedback on the disassembly of bonds, the model given by (7) reduces to the CIR process, see [?,?,?]:

$$
\mathrm{d} N_{t}=\left(c+(r-d) N_{t}\right) \mathrm{d} t+\sqrt{2 a N_{t}} \mathrm{~d} B_{t},
$$

with $c>0, a>0$ and $r-d \in \mathbb{R}$. It is known for demographical processes that the diffusion limit of discrete branching processes with immigration results in such processes [?], and show a dichotomy behaviour. Depending on the parameters, the density either almost surely has values close to 0 , or almost surely reaches large values, leading to the almost sure arrest of the cell in our model. Simulations of this process are displayed in Figure 5 . Some general properties of the CIR process are displayed in Appendix D. In particular, its stationary probability density is represented in Figure 5 and shows the transition between both behaviours.

Time to reach $u$ : It is also possible to obtain information on the time to reach a given value. More precisely, one can get the Laplace transform of the first hitting time of any value, starting at a given point $[?, ?]$. It is not possible to proceed to its inversion analytically. Numerical inversions procedures exist, and some of them are compared in [?]. They do not always provide satisfactory 


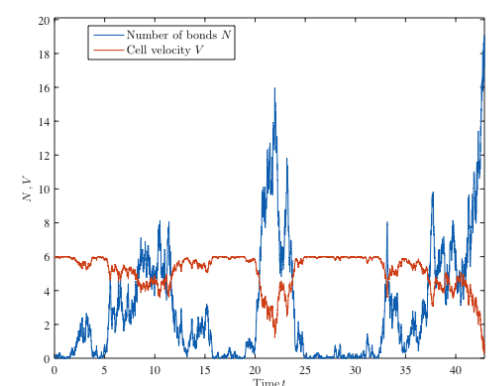

(a) Subcritical case with $(c, a, r, d)=(0.5,1.5,4.45,4.5)$.

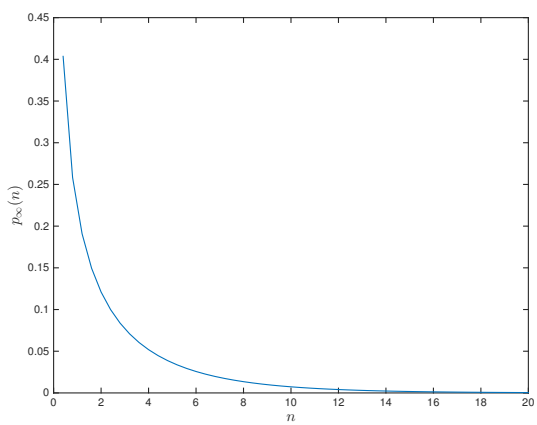

(c) Subcritical case with $(c, a)=(1,2)$.

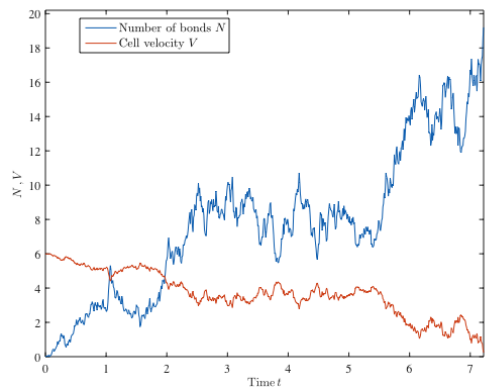

(b) Supercritical case with $(c, a, r, d)=(2,1,4,4)$.

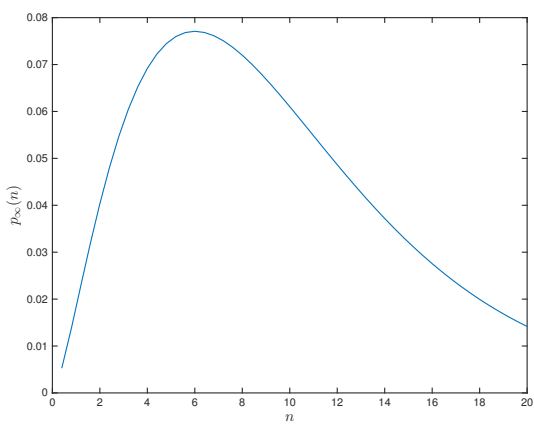

(d) Supercritical case with $(c, a)=(5,2)$.

Fig. 5: Up: numerical simulations of the CIR process (8). In the subcritical case, the adhesion density almost surely reaches zero, while in the supercritical case, an adhesive interaction is almost surely sustained. Down: numerical simulations of the stationary probability density of the CIR process for $(u, \alpha, r, d)=(20,0.1,4,4.5)$.

results: the integral of the output may not be equal to one, and negative values may appear. The procedure proposed by [?] seems satisfactory in this viewpoint.

In this paper, we follow the work of $[?, ?]$ to compute numerically the first hitting time density using an eigenfunction decomposition, following an approach used for diffusions $[?, ?, ?]$. For the CIR process, it is established in [?,?] that the same type of decomposition holds. We give now the result of [?] that provides a series expansion for the density $f_{T_{x \rightarrow y}}$ of the first hitting time of $y$ starting from $x$.

\section{Proposition 3 [?]}


i) For $0<x<y \in I$, and $t>0$ we have

$$
f_{T_{x \rightarrow y}}(t)=\sum_{n=1}^{+\infty} o_{n} \lambda_{n} e^{-\lambda_{n} t}
$$

with uniform convergence on $\left[t_{0},+\infty\right), t_{0}>0$, and $\left(\lambda_{n}\right)_{n}$ a strictly positive and strictly increasing sequence with $\lambda_{n}$ growing to $+\infty$ as $n$ goes to infinity. More precisely, we have that

$$
\lambda_{n}=(r-d) s_{n},
$$

with $\left(s_{n}\right)_{n}$ the strictly decreasing sequence of strictly negative roots of $\Phi(\cdot ; c / a ; \bar{y})=0$, with $\Phi\left(w_{1} ; w_{2} ; w_{3}\right)$ denotes the Kummer confluent hypergeometric function. The sequence $\left(o_{n}\right)_{n}$ is defined by

$$
o_{n}=-\frac{\Phi\left(s_{n} ; c / a ; \bar{x}\right)}{s_{n} \partial_{s}\left(\Phi\left(s_{n} ; c / a ; \bar{y}\right)\right)},
$$

for $\bar{y}:=-\frac{r-d}{a} y$ and $\bar{x}:=-\frac{r-d}{a} x$.

ii) Moreover, the following asymptotics hold:

$$
\lambda_{n} \underset{n \rightarrow+\infty}{\sim} \frac{(d-r) \pi^{2}}{4 \bar{y}}\left(n+\frac{c}{2 a}-\frac{3}{4}\right)^{2}-\frac{(r-d) c}{2 a},
$$

as well as

$$
\begin{array}{r}
o_{n \rightarrow+\infty} \frac{(-1)^{n+1} 2 \pi(n+c /(2 a)-3 / 4)}{\pi^{2}(n+c /(2 a)-3 / 4)^{2}-\frac{2 c}{a} \bar{y}} \times e^{\frac{1}{2}(\bar{x}-\bar{y})}\left(\frac{\bar{x}}{\bar{y}}\right)^{\frac{1}{4}-\frac{c}{2 a}} \\
\cos \left(\pi\left(n+\frac{c}{2 a}-\frac{3}{4}\right) \sqrt{\frac{\bar{x}}{\bar{y}}}-\frac{\pi c}{2 a}+\frac{\pi}{4}\right) .
\end{array}
$$

Therefore, the proposed numerical method requires to compute the set of negative roots of $\Phi$ to get approximations of the families $\left\{\lambda_{n}\right\}_{n}$ and $\left\{o_{n}\right\}_{n}$. The choice of the level of truncation for the approximation of $(9)$ can be made using the following estimate:

$$
\left|o_{n} \lambda_{N} e^{-\lambda_{N} t_{0}}\right| \underset{N \rightarrow+\infty}{\sim} A N e^{-B N^{2} t_{0}},
$$

for

$$
A=\frac{2 a \pi}{4 y} e^{\frac{\bar{x}-\bar{y}}{2}}\left(\frac{x}{y}\right)^{\frac{1}{4}-\frac{c}{2 a}}, \quad B=\frac{a \pi^{2}}{4 y} .
$$

Linetsky also notices that using (10)-11 instead of computating zeros of the Kummer function provides quite satisfactory results, in particular for $c / a$ small. For a better accuracy, one can also use the exact expression for the first term of the decomposition, then the estimates for the others. The following numerical simulation was performed using only the asymptotic expansion of 


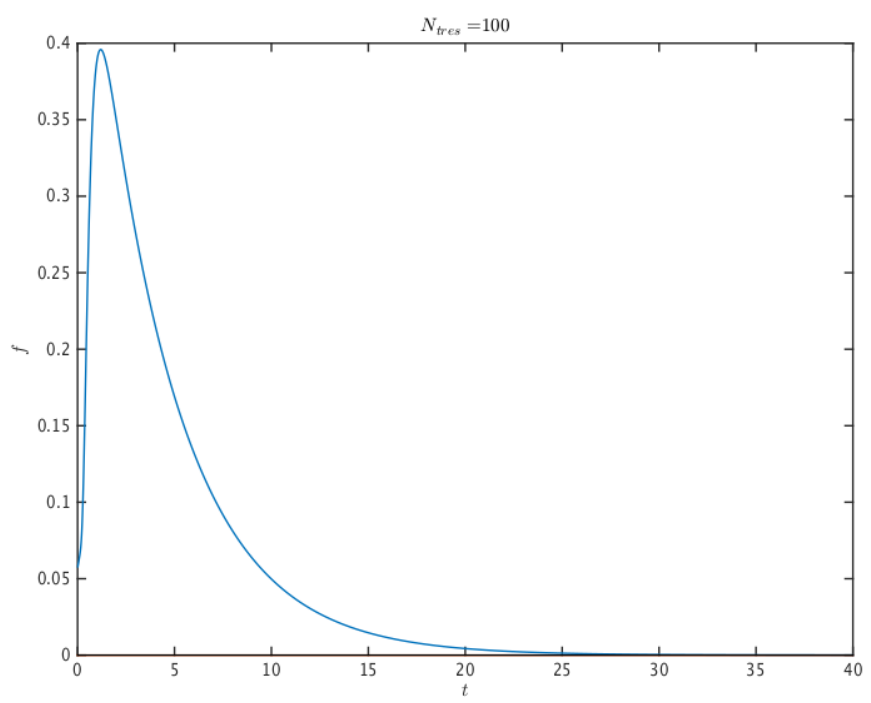

Fig. 6: Numerical simulation of an approximation of the asymptotic spectral decomposition of (9), the probability density of the first hitting time of 1 of a CIR process starting at 0.01. Parameters: $\Delta t=0.01, c=0.45, a=0.5$, $r=0.2$ and $d=1$. The sum is truncated at $N_{\text {tres }}=100$.

$\lambda_{n}$ and $o_{n}$ even for $n$ small, since it is observed that this approximation does not change qualitatively the profile (see Figure 6). Obviously, in this case, the obtained function is not a probability density, and an inconsistency tends to appear near $t=0$ due to the approximation, but the overall shape was conserved.

The general case Let us now focus on the general case of Equation (7). As a first approach we can use the 1D comparison principle (see e.g [?]) to compare the process with CIR processes. In this work, we follow another method and derive from (7) a Fokker-Planck equation on $p(n, t):=p\left(n, t \mid n_{0}, t_{0}\right)$ the probability density of $\left(N_{t}\right)_{t}$ conditionally to its initial condition. We obtain the following equation:

$$
\frac{\partial p(n, t)}{\partial t}=\frac{\partial}{\partial n} \underbrace{\left(-b(n) p(n, t)+\frac{1}{2} \frac{\partial}{\partial n}\left(\sigma^{2}(n) p(n, t)\right)\right)}_{\mathbf{J}(n, t)},
$$

where we recall that $b(n)=c+(r(n)-d(n)) n$, while $\sigma(n)=\sqrt{2 a n}$ and $\mathbf{J}(n, t)$ is the associated probability current. The natural boundary conditions are the 
following:

$$
\begin{aligned}
\mathbf{J}(0, t) & =0, \\
\lim _{n \rightarrow+\infty} p(n, t) & =0, \\
p(n, 0) & =\delta_{n=n_{0}} .
\end{aligned}
$$

We are interested in the mean time necessary for the process to reach the value $u$ starting from $n_{0} \in(0, u)$, that we denote by $\tau_{u}\left(n_{0}\right)$. This question can be adressed by considering the Fokker-Planck equation on $(0, u)$ with 0 a reflecting and $u$ an absorbing barrier. We show the following proposition.

Proposition 4 The mean time $\tau_{u}\left(n_{0}\right)$ necessary for the process to reach the value $u$ starting from $n_{0} \in(0, u)$ writes

$$
\tau_{u}\left(n_{0}\right)=\frac{1}{a} \int_{n_{0}}^{u} \int_{0}^{y}\left(\frac{z}{y}\right)^{\frac{c}{a}} z^{-1} e^{\frac{r}{a}(z-y)} \exp \left(\frac{d}{a \alpha} e^{\alpha u}\left(e^{-\alpha z}-e^{-\alpha y}\right)\right) \mathrm{d} z \mathrm{~d} y .
$$

Proof Write $G\left(n_{0}, t\right)$ the probability that a particle starting at $n_{0}$ is still in $(0, u)$ at time $t$. Then,

$$
G\left(n_{0}, t\right)=\int_{0}^{u} p\left(n, t \mid n_{0}, 0\right) \mathrm{d} n=\mathbb{P}\left(\tau_{u} \geq t\right) .
$$

Since the dynamics is homogeneous in time, we deduce that $p\left(n, t \mid n_{0}, 0\right)=$ $p\left(n, 0 \mid n_{0},-t\right)$ and $n_{0} \mapsto p\left(n, t \mid n_{0}, 0\right)$ satisfy the backward Fokker-Planck equation:

$$
\frac{\partial p\left(n, t \mid n_{0}, 0\right)}{\partial t}=b\left(n_{0}\right) \frac{\partial}{\partial n_{0}} p\left(n, t \mid n_{0}, 0\right)+\frac{1}{2} \sigma^{2}\left(n_{0}\right) \frac{\partial}{\partial n_{0}^{2}} p\left(n, t \mid n_{0}, 0\right),
$$

and $\left(n_{0}, t\right) \mapsto G\left(n_{0}, t\right)$ satisfies

$$
\frac{\partial G\left(n_{0}, t\right)}{\partial t}=b\left(n_{0}\right) \frac{\partial}{\partial n_{0}} G\left(n_{0}, t\right)+\frac{1}{2} \sigma^{2}\left(n_{0}\right) \frac{\partial}{\partial n_{0}^{2}} G\left(n_{0}, t\right) .
$$

The initial and boundary conditions are the following:

$$
\begin{aligned}
G\left(n_{0}, 0\right) & =\int_{0}^{u} \delta_{n-n_{0}} \mathrm{~d} n=\mathbb{1}_{[0, u]}\left(n_{0}\right), \\
\frac{\partial}{\partial n_{0}} G(0, t) & =0 \\
G(u, t) & =0 .
\end{aligned}
$$

Take $f \in \mathcal{C}^{1}\left(\mathbb{R}, \mathbb{R}_{+}\right)$non-decreasing. Then, classically, $\mathbb{E}\left[f\left(\tau_{u}\right)\right]=\int_{0}^{+\infty} f^{\prime}(t) \mathbb{P}\left(\tau_{u}>\right.$ $t) \mathrm{d} t=\int_{0}^{+\infty} f^{\prime}(t) G\left(n_{0}, t\right) \mathrm{d} t$. Hence, we get for $k>1$,

$$
\begin{aligned}
\tau_{u}\left(n_{0}\right) & =\mathbb{E}\left[\tau_{u}\right]=\int_{0}^{+\infty} G\left(n_{0}, t\right) \mathrm{d} t, \\
\tau_{u}^{k}\left(n_{0}\right) & =\mathbb{E}\left[\tau_{u}^{k}\right]=k \int_{0}^{+\infty} t^{k-1} G\left(n_{0}, t\right) \mathrm{d} t .
\end{aligned}
$$


By integration of 13 in time, we get the following ODEs on the family $\left(\tau_{k}\right)_{k \geq 1}$ :

$$
\left\{\begin{array}{l}
b\left(n_{0}\right) \tau_{u}^{\prime}\left(n_{0}\right)+\frac{1}{2} \sigma^{2}\left(n_{0}\right) \tau_{u}^{\prime \prime}\left(n_{0}\right)=-1, \\
\tau_{u}^{\prime}(0)=0 \\
\tau_{u}(u)=0
\end{array}\right.
$$

and for $k>1$,

$$
\left\{\begin{array}{l}
b\left(n_{0}\right) \tau_{u}^{k^{\prime}}\left(n_{0}\right)+\frac{1}{2} \sigma^{2}\left(n_{0}\right) \tau_{u}^{k^{\prime \prime}}\left(n_{0}\right)=-k \tau_{u}^{k-1}\left(n_{0}\right) \\
\partial_{n_{0}} \tau_{u}^{k}(0)=0 \\
\tau_{u}^{k}(u)=0
\end{array}\right.
$$

By direct integration, we can directly solve (14), allowing then to solve successively the problems (15). Write

$$
\Psi\left(n_{0}\right)=e^{\int_{0}^{n_{0}} \frac{2 b\left(n^{\prime}\right)}{\sigma^{2}\left(n^{\prime}\right)} \mathrm{d} n^{\prime}} .
$$

Then, we have

$$
\tau_{u}\left(n_{0}\right)=2 \int_{n_{0}}^{u} \frac{1}{\Psi(y)} \int_{0}^{y} \frac{\Psi(z)}{\sigma^{2}(z)} \mathrm{d} z \mathrm{~d} y .
$$

In practice, denoting $\epsilon>0$ for the lower bound in the integral instead of zero, we find that

$$
\Psi\left(n_{0}\right)=\left(\frac{n_{0}}{\epsilon}\right)^{\frac{c}{a}} \exp \left(\frac{r}{a} n_{0}-\frac{d}{\alpha \gamma a} e^{\alpha u}\left(1-e^{-\alpha \gamma n_{0}}\right)\right) .
$$

Explicit computations lead to the result.

We perform numerical simulations of $\tau_{u}(0)$ for varying values of the blood flow velocity $u$ (see Figure 7). Below a treshold flow velocity, the particle stops very fast, while it gets extremely slow above the treshold. The numerical phase plane shows a natural dependency on the adhesion creation rate $c$. The value of $a$, quantifying the noise intensity, has a direct effect on the range of the time of arrest, but do not change qualitatively the phase plane.

\section{Discussion}

In this work, we have presented a discrete model for ligands binding on artery walls. The model is based on a stochastic description of the formation of weak bonds between the cell and the adhesive molecules on the wall, and of the stronger ones arising by self-reinforcement. This phenomenon is modelled by a stochastic birth-and-death-type model. The binding dynamics is affected by the cell velocity by an interaction on their breaking rate: faster cells have shorter-lived bonds with the wall. 


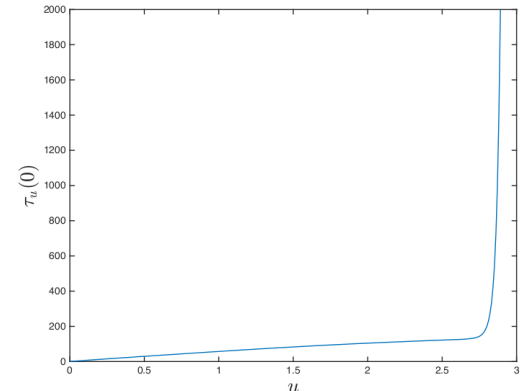

(a) Mean first stopping time $\tau_{u}(0)$ as a function of $u$.

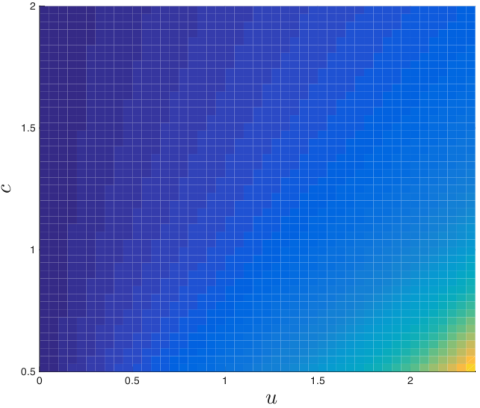

(b) Phase plane with respect to $u$ and the spontaneous binding rate $c$.

Fig. 7: Numerical simulations of the mean stopping time $\tau_{u}(0)$ defined by 12 as a function of $u$ (left), and phase plane depending on the blood velocity $u$ and the spontaneous binding rate $c$. Parameters: $\alpha=0.8, r=0.6, d=0.7$, $a=0.1$

4.1 Arrest and extravasation of circulating cells: interpreting the results of the model

The purpose of this model is to explore the events which follow the first wall contact and may determine whether the cell stops or goes back into the blood stream. More precisely, we are interested in the stopping time of the cell, that amounts to the first hitting time of a treshold value for the density of bonds. For this purpose, we derive continuous deterministic and stochastic models by performing some scaling limits.

In this framework, the growth in the number of bonds corresponds to cell deceleration. Eventually, in real vessels, the number of bonds becomes sufficiently large that circulating cells undergo firm arrest and extravasation. Once this happens, our model ceases to be valid; a different model is required to capture the fundamental processes involved in extravasation. Cell firm adhesion on the vessel wall have different implications depending on the cell type. In the context of Circulating Tumor Cells (CTCs), cell firm adhesion to the vessel wall is a step made towards the growth and formation of secondary tumors [?]. During the immune response, leukocytes carried by the blood flow experience arrest and possible firm adhesion to the vessel wall at sites related to inflammation and infection. Their extravasation then allows them to pursue their immune function. Let us also mention that researches are currently led on the development of drug delivery systems mimicking this phenomena. Overall, cell adhesion to vessel walls is a phenomenon showing major applications in biology and medecine. It is now clear that understanding the processes involved in the determination of the location of cell arrest is of prime importance. This 
justifies the development of new mathematical models able to explain the experimental observations.

4.2 Linear mean ODE model (3) does not agree with known observations

Our first finding concerns the mean linear ODE model in which there is no feedback of the cell velocity on the bond lifetime, namely ligand binding and dissociation rates are constant. In such a case, as expected, in the long-time limit, the mean number of bonds is independent of the shear velocity $u$. Such a result does not agree with in vitro and in vivo experiments, [?,?], which show that preferential regions where cells stop are those with a low flow velocity. Hence modelling this phenomenon requires a nonlinear model as the one we further studied.

4.3 The nonlinear model agrees qualitatively with known observations: hemodynamic forces affect the adhesion dynamics of circulating cells [?]

The study of the nonlinear model was done using a renormalization procedure based on the biological observation that the orders of magnitude between the adhesion dynamics and the cell motion are different. This allows us to rigorously build continuous models either deterministic or stochastic satisfied by the continuous number of bonds (or equivalently cell velocity).

The deterministic limit was obtained by considering a larger number of smaller links, and by accelerating also the rate of formation or encounter of these links (creation). In such a case, the reproduction, that accounts for the local reinforcement of the adhesion (the clustering), is not accelerated. Roughly speaking this means that the adhesion process is more "continuous" at the beginning, but the adhesion remains relatively weak. This could correspond to the description of rapid and reversible interactions of selectins with their carbohydrate ligands. In this case, we obtain a nonlinear ODE, for which the stationary states and their stability are given. It allows to outline the existence of a dichotomy behaviour in which the stability of the cell arrest is explicitly related to the balance between the blood flow velocity and the adhesion dynamics. It leads to the identification of a wall shear stress value that separates arrest from moving state. This is due to the fact that in this nonlinear model, the number of bonds is regulated by shear and may fall below one. This agrees with the known correlation between cell arrest location and blood flow [?].

In the case of the stochastic limit, the local reinforcement is also accelerated by the scaling, yielding a stronger adhesion with a nontrivial effect of the clustering on the stochastic fluctuations of the cell dynamics. As a consequence, at the limit, we obtain a diffusive SDE on the adhesion density that carries a nontrivial noise term. In the linear case where no interaction or feedback is described, the model resumes to a CIR process, for which some properties of first 
hitting times are known. We use a spectral method to perform numerical simulations of the corresponding probability density. Finally, for the full nonlinear model with interaction, we use a Fokker-Planck approach to derive an integral formulation of the mean arrest time of a cell. Numerical simulations of this quantity as a function of the flow velocity and the bonds creation rate show interesting results. First, a dichotomy appears in relation with the flow velocity: for low flows, the particle is stopped very fast, while above some treshold, the mean time to stop grows very steeply. Next, we checked that the range of the arrest time was mostly sensitive to the stochastic fluctuations intensity. This is in agreement with the observations stated in the introduction.

A second outcome is the ability of the nonlinear model to predict cell adhesion bistability. This follows from the competition between the two processes taking place in the cell-wall contact area: bond formation and rupture. This bistability could explain the variability of cells that stop in areas where the shear is neither too weak nor too strong.

Finally, this model gather key components involved in circulating cells behaviours in blood vessels. It allows to study how the blood flow affect the ability of cells to initiate an adhesive interaction with endothelial cells and to further form clusters of bonds. All in all, these phenomena mediate either the stable adhesion to the wall or the release of cells in the blood flow.

\subsection{Perspectives}

Further improvements would consist in extending the modelling framework in several directions. First, the model could take into account time-dependent rates, in order to consider a variable blood flow velocity, illustrating the effect of the heart cycle on the adhesion dynamics. Moreover, it is natural to extend the model to a 2D setting, where the vessel wall is a surface. For that purpose, adhesion proteins need to be described with respect to their location of the cell surafce. This situation could be handled by adding a spatial structure to the population of bonds, following the framework of [?,?] and further works on measure-valued stochastic processes.

Finally, further works should emphasize on the comparison with experimental measures to enlighten conditions of cell stopping. In particular, in [?], the authors study Circulating Tumor Cells (CTCs) in vivo, and keep track of the site of arrest of cells with respect to a tuned hemodynamic flow velocity. They show that cells arrest in blood vessels occurs at sites with permissive flow profiles. Since CTCs are large and quite rigid cells, our assumption of pointparticle cells is particularly relevant in this setting. Moreover, the adhesion molecules density on the vessel wall is also controlled experimentally, so that each feature of the model is characterized by experimental data. In this perspective, confronting the model to these data is of great interest, and could be complemented with the theoretical study of the distribution of positions of arrest. 
Acknowledgements The authors are very grateful to V.C. Tran and R. Voituriez for very helpful discussions and suggestions.

\section{Appendices}

\section{A Mathematical properties of the rescaled processes}

We consider here the rescaled process $\left(Z_{t}^{K}\right)_{t}$ in a general case, where $K$ is fixed. The two sets of rates considered satisfy the following hypothesis:

Hypothesis 1 For all $z \in \mathbb{R}_{+}$,

$$
\begin{gathered}
0 \leq c^{K}(u) \leq K c(u) \leq K c, \quad 0 \leq r^{K} \leq r+K a, \\
0 \leq d^{K}(z) \leq d(z)+K a \leq d e^{\alpha u}+K a,
\end{gathered}
$$

where $a$ is a positive constant. In addition the disassembly rate $z \mapsto d^{K}(z)$ is continuous. The total formation and dissociation rate write

$$
\lambda^{K}(z)=c^{K}(u)+r^{K} z, \mu^{K}(z)=d^{K}(z) z .
$$

By construction, $\left(Z_{t}^{K}\right)_{t \geq 0}$ is also a Markov process, and for $\Phi: \mathbb{R}_{+} \rightarrow \mathbb{R}$ measurable bounded, its infinitesimal generator writes

$$
L^{K} \Phi(Z)=\lambda^{K}(K Z)\left[\Phi\left(Z+\frac{1}{K}\right)-\Phi(Z)\right]+\mu^{K}(K Z)\left[\Phi\left(Z-\frac{1}{K}\right)-\Phi(Z)\right] .
$$

We show the following proposition.

Proposition 5 (Moment, martingale property) Assume that there exists $p \geq 2$ such that $\mathbb{E}\left[\left(Z_{0}^{K}\right)^{p}\right]<+\infty$, and that the rates satisfy Assumption 1 , Then, one has

1. $\forall T>0$,

$$
\mathbb{E}\left[\sup _{t \in[0, T]}\left(Z_{t}^{K}\right)^{p}\right]<+\infty
$$

2. for all measurable function $\Phi: \mathbb{R}_{+} \rightarrow \mathbb{R}$ for which there exists $C$ such that $\forall z \in \mathbb{R}_{+},|\Phi(z)|+\left|L^{K} \Phi(z)\right| \leq C\left(1+z^{p}\right)$,

$$
\Phi\left(Z_{t}^{K}\right)-\Phi\left(Z_{0}^{K}\right)-\int_{0}^{t} L^{K} \Phi\left(Z_{s}^{K}\right) \mathrm{d} s
$$

is a càdlàg $\left(\mathcal{F}_{t}\right)_{t \geq 0}$-martingale starting from 0 . 
3. The process

$$
M_{t}^{K}=Z_{t}^{K}-Z_{0}^{K}-\int_{0}^{t} \frac{1}{K} c^{K}\left(K Z_{s}^{K}\right)+\left(r^{K}\left(K Z_{s}^{K}\right)-d^{K}\left(K Z_{s}^{K}\right)\right) Z_{s}^{K} \mathrm{~d} s
$$

is a càdlàg square-integrable martingale starting from 0 and of quadratic variation

$$
\left\langle M^{K}\right\rangle_{t}=\frac{1}{K} \int_{0}^{t}\left\{\frac{1}{K} c^{K}\left(K Z_{s}^{K}\right)+\left(r^{K}\left(K Z_{s}^{K}\right)+d^{K}\left(K Z_{s}^{K}\right)\right) Z_{s}^{K}\right\} \mathrm{d} s .
$$

Proof Although the proof is similar to proposition 2.7 in [?] we recall it here for clarity. In the following $C$ will denote a positive constant which value can change from line to line. Let $(\Omega, \mathcal{F}, \mathbb{P})$ be a probability space, $Z_{0}$ an integervalued random variable, and $M(\mathrm{~d} s, \mathrm{~d} w)$ an independent Poisson Point Measure on $\mathbb{R}_{+}^{2}$, of intensity measure $\mathrm{d} s \mathrm{~d} w$. Denote by $\left(\mathcal{F}_{t}\right)_{t>0}$ the canonical filtration generated by these objects. Then, we define the $\left(\overline{\mathcal{F}}_{t}\right)_{t>0}$-adapted càdlàg process $\left(Z_{t}^{K}\right)_{t \geq 0}$ as the solution of the following SDE: $\forall t \geq 0$,

$$
Z_{t}^{K}=Z_{0}^{K}+\int_{0}^{t} \int_{\mathbb{R}_{+}}\left(\mathbb{1}_{0 \leq w \leq \lambda^{K}\left(Z_{s^{-}}^{K}\right)}-\mathbb{1}_{\lambda\left(Z_{s^{-}}^{K}\right)<w \leq \lambda^{K}\left(Z_{s^{-}}^{K}\right)+\mu^{K}\left(Z_{s^{-}}^{K}\right)}\right) M(\mathrm{~d} s, \mathrm{~d} w) .
$$

This representation is classical (see e.g [?,?]). The Poisson jumps related to the measure are accepted or rejected thanks to the indicator functions. The variable $w$ is then used as an acceptance parameter in order to get the desired rates for each event. Now, writing that $\mathbb{P}-a . s$, for a positive and measurable test function $\Phi$,

$$
\begin{aligned}
& \Phi\left(Z_{t}^{K}\right)=\Phi\left(Z_{0}^{K}\right)+\int_{0}^{t} \int_{\mathbb{R}_{+}}\left[\left(\Phi\left(Z_{s^{-}}^{K}+1\right)-\Phi\left(Z_{s^{-}}^{K}\right) \mathbb{1}_{0 \leq w \leq \lambda^{K}\left(Z_{s^{-}}^{K}\right)}\right.\right.
\end{aligned}
$$

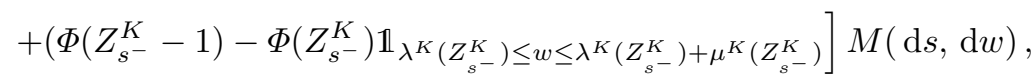

so that for $\Phi\left(Z_{t}^{K}\right)=\left(Z_{t}^{K}\right)^{p}$ and neglecting the negative death term, we get

$$
\left(Z_{t}^{K}\right)^{p} \leq\left(Z_{0}^{K}\right)^{p}+\int_{0}^{t} \int_{\mathbb{R}_{+}}\left(\left(Z_{s^{-}}^{K}+1\right)^{p}-\left(Z_{s^{-}}^{K}\right)^{p}\right) \mathbb{1}_{0 \leq w \leq \lambda^{K}\left(Z_{s^{-}}^{K}\right)} M(\mathrm{~d} s, \mathrm{~d} w) .
$$

Taking expectations, and using that for $p \in \mathbb{N},(1+z)^{p}-z^{p} \leq C(p)\left(1+z^{p-1}\right)$, we obtain that

$$
\begin{aligned}
\mathbb{E}\left[\sup _{t \in\left[0, T \wedge \tau_{u}\right]}\left(Z_{t}^{K}\right)^{p}\right] & \leq \mathbb{E}\left[\left(Z_{0}^{K}\right)^{p}\right]+C(p) \mathbb{E}\left[\int_{0}^{T \wedge \tau_{u}}\left(1+Z_{t^{-}}^{K}\right)\left(1+\left(Z_{t^{-}}^{K}\right)^{p-1}\right) \mathrm{d} t\right] \\
& \leq \mathbb{E}\left[\left(Z_{0}^{K}\right)^{p}\right]+C(p)\left(T+\int_{0}^{T} \mathbb{E}\left[\sup _{u \in\left[0, t \wedge \tau_{u}\right]}\left(Z_{u^{-}}^{K}\right)^{p}\right] \mathrm{d} t\right) .
\end{aligned}
$$


We conclude with the Gronwall Lemma together with the assumption on the initial condition. Point 2. of the proposition is a classical property of such Markov processes, and is used to obtain the other points. The quadratic variation is obtained by considering the semi-martingale structure for $\Phi(z)=z^{2}$ and $\Phi(z)=z$, and applying the Ito formula to the latter to obtain another semi-martingale structure for $\left(Z_{t}^{K}\right)^{2}$. The uniqueness of the decomposition leads to the result.

\section{B Proof of the deterministic convergence}

The proof is similar to the one in [?,?]. It is based on a compactness-uniqueness argument. First, note that the uniform estimate

$$
\forall T>0, \sup _{K>0} \mathbb{E}\left[\sup _{t \in\left[0, T \wedge \tau_{u}\right]}\left(Z_{t}^{K}\right)^{p}\right]<+\infty .
$$

holds, following the same framework as in Proposition 5 in Appendix A, since we have $K$-uniform bounds. This allows to show the uniform tightness of the sequence of laws $\left(Q^{K}\right)_{K}$ of $\left(Z^{K}\right)_{K}$.

Then, from the Prokhorov theorem we deduce the relative compactness of the family of laws $\left(Q^{K}\right)_{K}$ on $\mathbb{D}\left([0, T], \mathbb{R}_{+}\right)$. Consider a convergent subsequence of limit $Q$, and consider a corresponding sequence of processes converging in distribution to $n \in \mathbb{D}\left([0, T], \mathbb{R}_{+}\right)$of law $Q$. We need to identify this limit. Firstly, since the jumps of $\left(Z_{t}^{K}\right)_{t}$ are of the form $1 / K$, we know that any process of law $Q$ is almost surely strongly continuous. For $t \leq T \wedge \tau_{u}$, denote

$$
\Psi_{t}(n):=n_{t}-n_{0}-\int_{0}^{t}\left\{c(u)+\left(r-d\left(n_{s}\right)\right) n_{s}\right\} \mathrm{d} s .
$$

We can prove easily that for all $t \leq T, \mathbb{E}_{Q}\left[\left|\Psi_{t}(n)\right|\right]=0$. Finally, the convergence follows from the uniqueness in $\mathcal{C}\left([0, T], \mathbb{R}_{+}\right)$of the solution to $[6)$, which comes from the Lipschitz-continuity of the disassembly rate.

\section{Proof of the stochastic convergence}

C.1 Uniform estimates on the processes

We first prove two propositions that give uniform estimates on the process. They will be used to show that any sequence of laws associated with the sequence of processes $\left(Z_{t}^{K}\right)_{K}$ is tight.

Proposition 6 Consider the process $\left(Z_{t}^{K}\right)_{t \geq 0}$ defined in (4). If

$$
\sup _{K>0} \mathbb{E}\left[\left(Z_{0}^{K}\right)^{2}\right]<+\infty
$$

then for $T<+\infty$,

$$
\sup _{K} \sup _{t \in[0, T]} \mathbb{E}\left[\left(Z_{t}^{K}\right)^{2}\right]<+\infty
$$


Proof The infinitesimal generator associated with $\Phi(x)=x^{2}$ easily yields that

$$
L^{K} \Phi\left(Z_{s}^{K}\right) \leq\left(c+r Z_{s}^{K}\right)\left(2 Z_{s}^{K}+\frac{1}{K}\right)+2 a Z_{s}^{K} \leq C\left(1+Z_{s}^{K}+\left(Z_{s}^{K}\right)^{2}\right) .
$$

Hence, we can deduce using 16 that

$$
\mathbb{E}\left[\left(Z_{t}^{K}\right)^{2}\right] \leq \mathbb{E}\left[\left(Z_{0}^{K}\right)^{2}\right]+C\left(t+\int_{0}^{t} \mathbb{E}\left[Z_{s}^{K}\right]+\mathbb{E}\left[\left(Z_{s}^{K}\right)^{2}\right] \mathrm{d} s\right)
$$

Finally, since $\mathbb{E}\left[Z_{s}^{K}\right] \leq C\left(1+\mathbb{E}\left[\left(Z_{t}^{K}\right)^{2}\right]\right)$, by the Gronwall lemma, there exists a constant $C(T)$ such that $\mathbb{E}\left[\left(X_{t}^{K}\right)^{2}\right] \leq C(T)$, hence the result.

Proposition 7 Consider the process $\left(Z_{t}^{K}\right)_{t \geq 0}$ defined in (4) for all $K>0$. Assume that $\sup _{K>0} \mathbb{E}\left[\left(Z_{0}^{K}\right)^{2}\right]<+\infty$. Then, for $T<+\infty$,

$$
\sup _{K} \mathbb{E}\left[\sup _{t \in[0, T]} Z_{t}^{K}\right]<+\infty
$$

Proof From (17), we write that

$$
\sup _{t \in[0, T]} Z_{t}^{K} \leq \sup _{t \in[0, T]}\left|M_{t}^{K}\right|+Z_{0}^{K}+c T+r \int_{0}^{t} Z_{s}^{K} \mathrm{~d} s .
$$

Now, by the Burkholder-Davis-Gundy inequality,

$$
\mathbb{E}\left[\sup _{t \in[0, T]}\left|M_{t}^{K}\right|\right]^{2} \leq \mathbb{E}\left[\sup _{t \in[0, T]}\left|M_{t}^{K}\right|^{2}\right] \leq 4 \mathbb{E}\left[\left|M_{T}^{K}\right|^{2}\right]=4 \mathbb{E}\left[\left\langle M^{K}\right\rangle_{T}\right]
$$

and as $\mathbb{E}\left[Z_{0}^{K}\right]<+\infty$,

$$
\mathbb{E}\left[\sup _{t \in[0, T]} Z_{t}^{K}\right] \leq 2 \mathbb{E}\left[\left\langle M^{K}\right\rangle_{T}\right]^{1 / 2}+C(T)+r \mathbb{E}\left[\int_{0}^{t} Z_{s}^{K} \mathrm{~d} s\right]
$$

We use 18 to get

$$
\mathbb{E}\left[\left\langle M^{K}\right\rangle_{T}\right] \leq c T+\left(r+d e^{\alpha u}+2 a\right) \int_{0}^{t} \mathbb{E}\left[Z_{s}^{K}\right] \mathrm{d} s \leq C(T)
$$

thanks to Proposition 6. We conclude using the Gronwall lemma. 
C.2 Convergence

The proof follows the same outline as in the deterministic case. First, we can prove similarly that the sequence of laws $\left(Q^{K}\right)_{K}$ of the processes $\left(Z^{K}\right)_{K}$ is uniformly tight in $\mathcal{L}\left(\mathbb{D}\left([0, T], \mathbb{R}_{+}\right)\right)$. Indeed, denote $\left(A_{t}^{K}\right)_{t \geq 0}$ the finite variation process associated to $\left(Z_{t}^{K}\right)_{t}$. The Aldous and Rebolledo criterion [?] states that we need to prove that for all $T>0$ the following inequalities hold true:

1.

$$
\sup _{K>0} \mathbb{E}\left[\sup _{t \in[0, T]}\left|Z_{t}^{K}\right|\right]<+\infty .
$$

2. $\forall \varepsilon>0, \forall \eta>0, \exists \delta>0, K_{0} \in \mathbb{N}^{*}$ such that for all sequence $\left(\sigma_{K}, \tau_{K}\right)_{K}$ of stopping times with $\sigma_{K} \leq \tau_{K} \leq T$,

(a)

$$
\sup _{K \geq K_{0}} \mathbb{P}\left(\left|<M^{K}>_{\tau_{K}}-<M^{K}>_{\sigma_{K}}\right| \geq \eta, \tau_{K} \leq \sigma_{K}+\delta\right) \leq \varepsilon,
$$

(b)

$$
\sup _{K \geq K_{0}} \mathbb{P}\left(\left|A_{\tau_{K}}^{K}-A_{\sigma_{K}}^{K}\right| \geq \eta, \tau_{K} \leq \sigma_{K}+\delta\right) \leq \varepsilon .
$$

It follows from direct computations, Proposition 7, and the Markov inequality. Here we aim at identifying the limiting values. For $Y=\left(Y_{t}\right)_{t \geq 0} \in \mathbb{D}\left([0, T], \mathbb{R}_{+}\right)$, define

$$
\widetilde{M}_{t}(Y)=Y_{t}-Y_{0}-\int_{0}^{t}\left\{c(u)+\left(r-d\left(Y_{s}\right)\right) Y_{s}\right\} \mathrm{d} s .
$$

We need to show that $\widetilde{M}_{t}(N)$ is a twice-integrable continuous martingale with quadratic variation process defined by

$$
\langle\widetilde{M}\rangle_{t}=2 a \int_{0}^{t} Y_{s} \mathrm{~d} s
$$

First, we show that $\widetilde{M}(N)$ is a martingale. Take $0 \leq s_{1}<\ldots<s_{n}<s<t$, and $\Phi_{1}, \ldots, \Phi_{n}$ continuous bounded functions from $\mathbb{R}$ to $\mathbb{R}$. Define $\Psi: \mathbb{D}([0, T], \mathbb{R}) \rightarrow$ $\mathbb{R}$ by

$$
\Psi(Y)=\Phi_{1}\left(Y_{s_{1}}\right) \ldots \Phi_{n}\left(Y_{s_{n}}\right)\left[Y_{t}-Y_{s}-\int_{s}^{t}\left\{c(u)+\left(r-d\left(Y_{s}\right)\right) Y_{s}\right\} \mathrm{d} u\right]
$$

We can show that $\mathbb{E}[\Psi(N)]=0$, as in the proof of Theorem 1 . The new argument in this proof consists in showing that the bracket of $\widetilde{M}$ is given by (20).

1. First, consider the $K$-dependent semimartingale obtained from 16 with $\Phi\left(Z^{K}\right)=\left(Z^{K}\right)^{2}$, that is related to the generator:

$$
\begin{aligned}
L^{K} \Phi\left(Z_{s}^{K}\right) & =2 Z_{s}^{K}\left(c(u)+\left(r-d\left(Z_{s}^{K}\right)\right) Z_{s}^{K}\right) \\
& +\frac{1}{K}\left(c(u)+\left(r+d\left(Z_{s}^{K}\right)+2 K a\right) Z_{s}^{K}\right) .
\end{aligned}
$$


We can show that we have at the limit the following martingale:

$$
\tilde{N}_{t}=\left(N_{t}\right)^{2}-\left(N_{0}\right)^{2}-\int_{0}^{t}\left\{2 N_{s}\left(c(u)+\left(r-d\left(N_{s}\right)\right) N_{s}\right)+2 a N_{s}\right\} \mathrm{d} s .
$$

2. Moreover, applying the Itô formula to 19 shows that

$$
N_{t}^{2}-N_{0}^{2}-<\widetilde{M}>_{t}-\int_{0}^{t} 2 N_{s}\left(c(u)+\left(r-d\left(N_{s}\right)\right) N_{s}\right) \mathrm{d} s
$$

is a martingale. We conclude by the uniqueness of the semimartingale decomposition of $N_{t}^{2}$.

The equivalence between the obtained martingale problem and the SDE (7) is a consequence of the classical martingale identification

$$
M_{t}=\int_{0}^{t} \sqrt{2 a N_{s}} \mathrm{~d} B_{s}
$$

see for example [?]. Finally, the pathwise uniqueness of the solution to this $\mathrm{SDE}$ is classical in 1D since the drift is Lipschitz-continuous and the diffusion coefficient is $1 / 2$-Hölder (see e.g [?]).

Remark 5 The solution is strong and has the strong Markov property.

\section{The CIR process}

In this paragraph, we give some classical results about the CIR process. For more details we refer to $[?, ?]$.

Reaching zero

As mentioned in [?], for $c>0$ and a positive initial state, $\{N=0\}$ is naturally a reflective barrier for the process (8). We can also provide information about the probability to hit 0 , depending on the quantity $\delta:=\frac{2 c}{a}$, or equivalently on the comparison between $a$ and $c$ : denote $\mathbb{P}^{0}$ the probability to hit 0 in finite time, then one has

$$
\begin{array}{c|c|c}
\multicolumn{2}{c|}{c<a} & c \geq a \\
r-d \leq 0 & r-d>0 & \\
\hline \mathbb{P}^{0}=1 & \mathbb{P}^{0} \in(0,1) & \mathbb{P}^{0}=0
\end{array}
$$

These results can be intuitively understood following the correspondance between CIR processes and Orstein-Uhlenbeck processes for $r-d<0$. Indeed, consider $D$ such processes $\left(X^{1}, \cdots, X^{D}\right)$ such that $\forall i=1, \cdots, D$,

$$
\mathrm{d} X_{t}^{i}=-\frac{1}{2} \beta X_{t}^{i} \mathrm{~d} t+\frac{1}{2} \sigma \mathrm{d} B_{t}^{i},
$$


with $\left(B^{i}\right)_{i} \in\{1, \cdots, D\}$ independent Brownian motions and $\beta>0$. Each process follows a stochastic dynamics that is drifted to zero. Now, similarly as for Brownian motion, for $D=1$, the process almost surely hits zero infinitely many times, while for $D \geq 2$, it has a null probability of reaching zero even once.

Using the Itô formula, it is possible to write the SDE that verifies the squared euclidean norm of $\left(X^{1}, \cdots, X^{D}\right)$, that is to say $R=\left(X^{1}\right)^{2}+\cdots+$ $\left(X^{D}\right)^{2}$ : we have

$$
\mathrm{d} R_{t}=\left(\frac{\sigma^{2} D}{4}-\beta R_{t}\right) \mathrm{d} t+\sigma \sqrt{R(t)} \mathrm{d} B_{t},
$$

with $B$ a Brownian motion. Then, denoting $D=4 c / \sigma^{2}>0$, where $\sigma^{2}=2 a$, it is possible to derive equation (8) with $r-d=-\beta \leq 0$. Therefore, if $D$ is an integer, the CIR process admits a representation as the squared norm of $D$ Ornstein-Uhlenbeck processes. More generally, the CIR process relates rigorously to the Squared Radial Ornstein-Uhlenbeck process, as it will be precised below.

Distribution

It is known that for $\delta=\frac{2 c}{a} \in \mathbb{N}$ and $r-d<0$, we have

$$
N_{t} \mid n_{0}=\frac{\left(1-e^{(r-d) t}\right) 2 a}{2(d-r)} Y_{t}
$$

with $Y_{t}$ following a non-central chi-square distribution with $\delta$ degrees of freedom, and a non-centrality parameter $\xi_{t}=n_{0} \frac{2(d-r)}{\left(1-e^{(r-d) t}\right) a} e^{(r-d) t}$. This also results from the representation as the squared euclidean norm of a $\delta$-dimensional Ornstein-Uhlenbeck process. The integral form of the SDE (8) allows to compute the mean solution directly, and the variance of the solution (using additionally the Ito formula). We compute

$$
\begin{aligned}
\mathbb{E}\left[N_{t} \mid n_{0}\right] & =n_{0} e^{(r-d) t}+\frac{c}{d-r}\left(1-e^{(r-d) t}\right) \\
\operatorname{Var}\left(N_{t} \mid n_{0}\right) & =n_{0} \frac{2 a}{d-r}\left(e^{(r-d) t}-e^{2(r-d) t}\right)+\frac{a c}{(d-r)^{2}}\left(1-e^{(r-d) t}\right)^{2} .
\end{aligned}
$$

The corresponding probability density $p_{n_{0}}$ writes for $n \geq 0, n_{0}>0$ and $\kappa_{t}=\frac{d-r}{\left(1-e^{(r-d) t}\right) a}$ :

$$
p_{n_{0}}\left(n ; k, \xi_{t}\right)=\kappa_{t}\left(\frac{n}{n_{0} e^{(r-d) t}}\right)^{\frac{c}{a}-1} e^{-\kappa_{t}\left[n_{0} e^{(r-d) t}+n\right]} I_{\frac{c}{a}-1}\left(2 \kappa_{t} \sqrt{n_{0} n e^{(r-d) t}}\right)
$$

where $I_{\alpha}$ is the modified Bessel function of the first kind whose definition we recall now:

$$
I_{\alpha}(x):=\Sigma_{m=0}^{\infty} \frac{1}{m ! \Gamma(m+\alpha+1)}\left(\frac{x}{2}\right)^{2 m+\alpha},
$$


where $\Gamma$ is the Gamma function $\Gamma(t)=\int_{0}^{\infty} x^{t-1} e^{-x} \mathrm{~d} x$.

A stationary distribution exists if and only if $r-d<0$ [?]. In that case, the previous approach gives a possible stationary probability density. Then, writing the Fokker-Planck equation associated to Equation (8), we can check that the stationary density writes

$$
p_{\infty}(n)=\left(\frac{d-r}{a}\right)^{\frac{c}{a}} \frac{1}{\Gamma\left(\frac{c}{a}\right)} n^{\frac{c}{a}-1} e^{\frac{r-d}{a} n} .
$$

It can be noticed that

$$
p_{\infty}(n)=\mathcal{N} e^{-\phi(n)}
$$

for $\phi(n)=\left(1-\frac{c}{a}\right) \ln (n)+\frac{d-r}{a} n$ the corresponding potential, and $\mathcal{N}$ a normalization constant. 